\title{
A dynamic post-evaluation analysis strategy based on Space syntax for optimizing middle school campus layout
}

\author{
Kexin Guo ${ }^{1}$, Guoqing Peng ${ }^{2 *}$, Jining Pang ${ }^{2}$, Shuaibing Shen ${ }^{2}$, Xuewen Lin ${ }^{2}$, Qian Wan ${ }^{2.3}$ \\ ${ }^{1}$ School of Information Engineering, China University of Geosciences (Beijing), Beijing 100083, China \\ ${ }^{2}$ Hualan Design and Consulting Group Company Ltd., Nanning 530011, China \\ ${ }^{3}$ School of Architecture and Transportation Engineering, Guilin University of Electronic Technology, Guilin 541004, China
}

\begin{abstract}
Space syntax has injected new vitality into quantitative and parameterized planning and design of middle school campus. Although, there are extensive interpretative problems in space syntax that need to be clarified during engineering planning and design of campus. This paper focuses on: (1) the parameters and models of space syntax are clarified from the perspectives of planning and design; (2) the specific application of space syntax in campus planning and design is discussed from the four stages of preliminary research, scheme analysis, scheme design and scheme optimization; (3) a relatively reliable quantitative, visual and procedural post-evaluation system of campus planning and design is provided with GIS spatial analysis technology and dynamic simulation means.
\end{abstract}

\section{INTRODUCTION}

With the development of campus planning, the attention is increasing significantly on space planning, culture shaping, ecological and sustainable developing. Therefore, it is a critical topic for quantitatively estimating the campus spatial form planning, predicting and evaluating the campus layout through with a dynamic post-evaluation strategy. As a means of spatial logic analysis, spatial syntax provides quantitative evaluation of campus layout through the relation between spatial organization and spatial function. However, on the one hand, the researchers are more on the university campus layout evaluation and less on middle school campus [1-2]; on the other hand, the space users' travel characteristics are usually ignored. The travel characteristics of middle school students show lower flexible than University students but higher than primary school students [3]. The rationality of middle school campus layout has an important impact on personal development and growth. Therefore, exploring the evaluation and analysis system of middle school campus construction layout is significant.
By combing domestic and foreign literature, the analyses are mostly carried out from the perspective of graph theory logic [4]. Planners have different understandings of the parameters, which hinders the space syntax application in practical work. Therefore, the research of space syntax in campus planning and design mostly stays at the level of static evaluation, and fails to provide constructive guidance and suggestions in the field of planning and design. It only finds and solves problems, and does not dynamically verify and improve the problems. This paper explored the students' behaviour law, established the space syntax model to interpret the syntax parameters from the perspective of planning and design, broke the understanding barriers between planning and design and space syntax. At the same time, combined the simulation technology of social force model to realize the improvement of planning and design evaluation from static to dynamic, verify the analysis conclusion and make dynamic adjustment, assist the scheme optimization, and establish a set of evaluation system for completed projects. The evaluation process is shown in Figure 1.

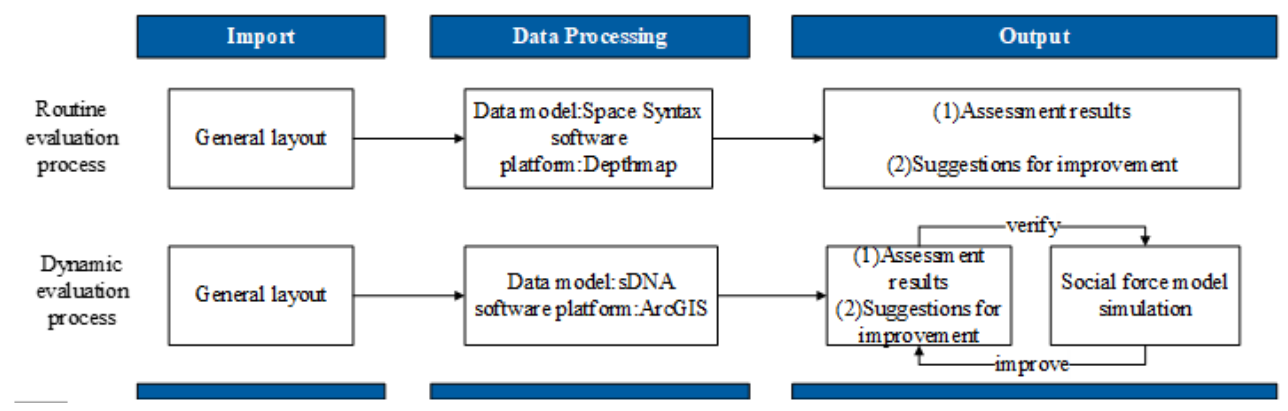

Figure 1. The difference between Routine evaluation process and Dynamic evaluation process.

\footnotetext{
* Corresponding author: 243564846@qq.com
} 


\section{METHODOLOGY}

Campus space is an important place for students to live on, which core is to provide convenient learning and communication space for students and enrich their campus life. In order to better guide the overall development of middle school campus and improve the space quality, the planning of campus space layout must change from qualitative design to quantitative and visualization design.

Researchers' exploration of space syntax has provided new development opportunities for quantitative planning and design, but interpret the space syntax indicators from the perspective of planning and design, and clearly explain the operation process and application methods in each stage of planning and design are needed.

This study developed to be expecting for based on the domestic and foreign achievements of secondary school campus planning and evaluation, establishes the specific application methods of spatial cognition, model building, spatial analysis and simulation optimization in turn from the four stages of preliminary investigation, scheme analysis, scheme design and scheme optimization. Combine with the practical case of Guangxi Nanning No.2 Middle School, explored the application of spatial syntax in campus planning and design, established a parametric, process, visualization planning and design paradigm, which can provide guidance for the subsequent middle school campus planning and design. The dynamic post-evaluation

\section{RESULTS \& DISCUSSION}

\subsection{First stage: preliminary investigation - spatial cognition}

The preliminary research stage is the basis and support of planning and design. Comprehensive understanding the regional relationship and geographical conditions of space is not only the basis of finding the right planning entry point, but also the premise of establishing the syntactic model. According to the spatial syntactic model, we can find out the cognitive rules of users and verify the accuracy of spatial cognition. Therefore, spatial cognition is the key to quantitative planning and design. Spatial cognition should not only consider the spatial structure, but also consider the relationship between users' daily living environment and users' travel behaviour. At present, most studies usually ignore the fact that users are the final decision makers of space, and lack of exploration on users' travel rules [1-2], which leads to the reduction of space experience and belonging sense, produces the unnecessary commuting distance.

This study summarizes the main middle school students' travel behaviours and their main travel characteristics (Table 1). According to the time table of middle school students, combined with typical activities such as recess activities, teaching place conversion, sightseeing, divided the 5-minute travel radius, 10minute travel radius and global radius; according to the convex space model, three topological radius and global step radius are divided, which lays the foundation for establishing the multi-scale syntactic model of middle school cam pus and analysing the service radius range of space.

Table 1.Typical travel behaviours in middle school campus

\begin{tabular}{|c|c|c|c|c|}
\hline $\begin{array}{c}\text { Travel } \\
\text { behavior }\end{array}$ & Scale & $\begin{array}{c}\text { Time } \\
\text {-limit }\end{array}$ & Space & Travel characteristics \\
\hline Studying & Big & Middle & $\begin{array}{c}\text { Dormitory - } \\
\text { Teaching zone }\end{array}$ & $\begin{array}{c}\text { Large flow of people per unit time; the road system requires } \\
\text { high walking capacity, and the space needs high openness }\end{array}$ \\
\hline Dining & Big & Middle & $\begin{array}{c}\text { Canteen - teaching / } \\
\text { living zone }\end{array}$ & $\begin{array}{c}\text { The gathering regularity of people flow is strong, showing } \\
\text { the form of small groups and the road needs higher carrying } \\
\text { capacity and high space openness }\end{array}$ \\
\hline Moving & Middle & Short & $\begin{array}{c}\text { Teaching area - } \\
\text { Experimental } \\
\text { Building }\end{array}$ & $\begin{array}{c}\text { The recess time is limited, the flow rate of people is fast, and } \\
\text { the road needs high accessibility }\end{array}$ \\
\hline activities & Small & Short & $\begin{array}{c}\text { Inside the teaching } \\
\text { area }\end{array}$ & $\begin{array}{c}\text { The target location is relatively scattered. It is easy to cause } \\
\text { cross conflict between students due to the inconsistency of } \\
\text { goals. Need high accessibility at small scale }\end{array}$ \\
\hline Gathering & Big & Short & $\begin{array}{c}\text { The pedestrian flow is relatively concentrated, and the } \\
\text { Teaching zone- } \\
\text { demand for pedestrian carrying capacity is large. High } \\
\text { accessibility should be ensured at all scales }\end{array}$ \\
\hline
\end{tabular}

Table 2. Interpretation of spatial syntactic parameters from the perspective of planning

\begin{tabular}{|ll|l|l|}
\hline Space Syntax & Planning and design \\
\hline Integration & $\begin{array}{l}\text { The high integration region expresses the Evaluate whether the space meets the attraction } \\
\text { agglomeration effect caused by pedestrian and demand and whether the road bearing capacity Functionality } \\
\text { vehicle flow under the travel radius }\end{array}$ & $\begin{array}{l}\text { meets the road grade } \\
\text { Choice }\end{array}$ & High degree of travel refers to that people getEvaluate whether the space meets the Accessibility \\
\hline
\end{tabular}



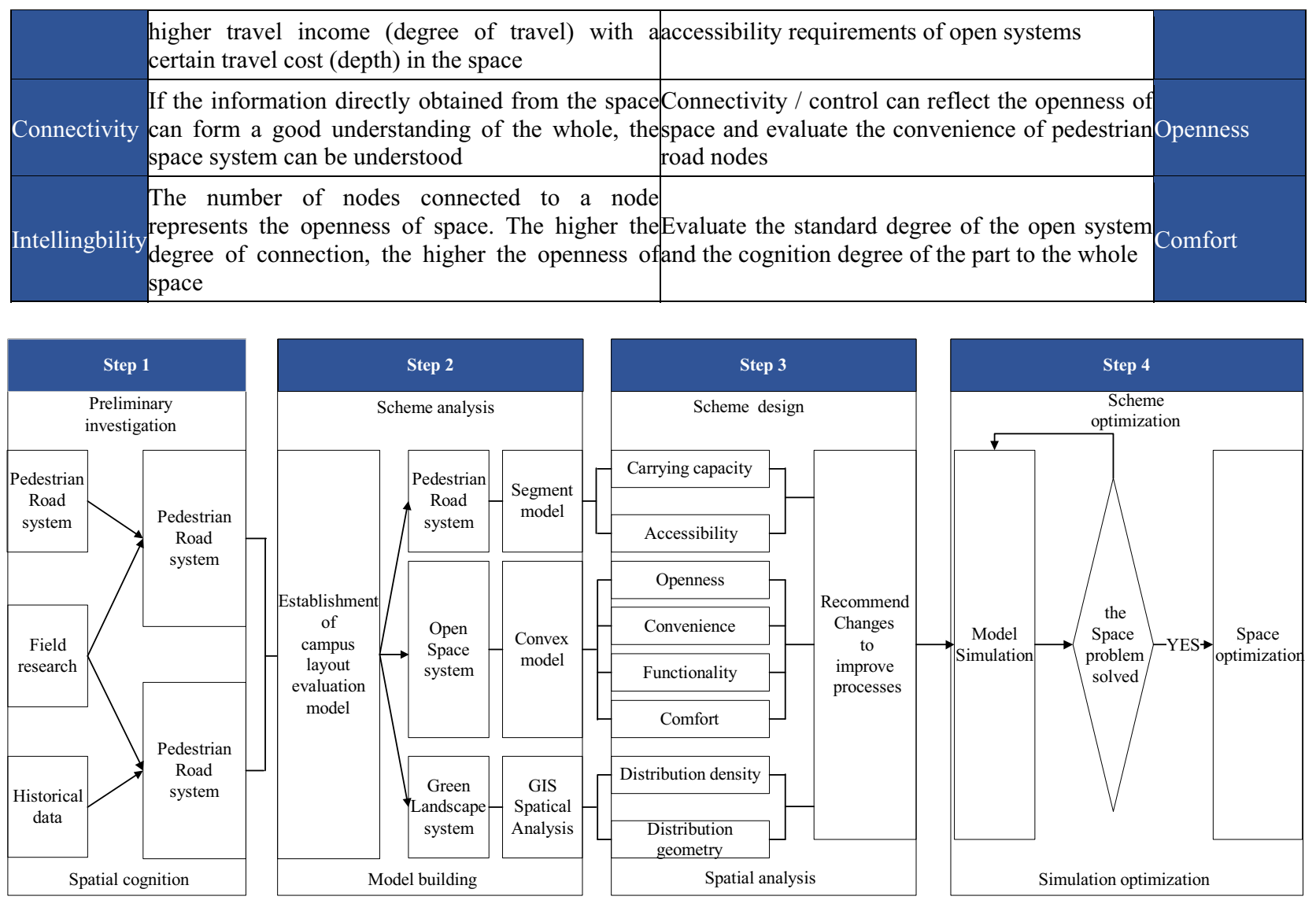

Figure 2. The dynamic post-evaluation system

\subsection{Second stage: scheme analysis - model building}

Middle school campus is similar to university campus in the space elements composition, so this study chose the open space system, pedestrian road system and green landscape system mentioned by academician Jingtang $\mathrm{He}$ [5] in Contemporary university campus planning theory and design practice to establish a space syntax analysis model. However, space syntax has a variety of parameters, it is difficult to be chosen by planners [6], this study from the perspective of planning, reinterpreted the space syntax parameters at the planning and design level, as shown in table 3. On this basis, combined with the spatial configuration of open space, pedestrian road system and green landscape system, the authors select parameter model and evaluate the space function.

\subsubsection{Open space system}

Open space is important for teachers and students to live and study, it is the top priority of campus space layout evaluation. The traditional space syntax analysis is not from the perspective of planning and design for the corresponding interpretation and analysis, nor can it evaluate the function of space in the whole. For example, as an image and functional building, the comprehensive building should have a higher space openness, but as a rest place, the dormitory building needs to meet the quiet and hidden needs, and should have a lower openness. Therefore, on the basis of parameter interpretation and functional characteristics analysis, this study established convex models of three topological space radius scale and global radius scale, and evaluates six typical functional areas (teaching area, complex building, dormitory area, canteen, leisure area and sports place) point-to-point with the parameters of integration, average depth, connectivity and comprehensibility.

\subsubsection{Pedestrian road system}

According to campus road function, it can be divided into two types: one is the main road connecting each partition, which carries the main traffic in the campus with high accessibility and high carrying capacity; the other is the connecting road inside each partition, which carries the traffic flow in the area with high accessibility. Therefore, the accessibility and carrying capacity of campus roads need to be matched with their spatial functions to avoid unnecessary commuting distance and road congestion. In this study, according to the characteristics of the linear elements of the road, combined with the spatial syntax line segment model, considering the deflection angle influence of the road network on the passenger flow, the campus line segment model is established in three scales: Micro (5min travel radius), meso (10min travel radius) and macro to evaluate the accessibility and carrying capacity of pedestrian road system in the campus planning and design. 


\subsubsection{Green landscape system}

Because there is no suitable analysis model, the analysis of green landscape system in campus is usually ignored in the space syntax evaluation. Based on the advantages of ArcGIS spatial analysis, this study integrated the core density analysis and standard deviation ellipse analysis of GIS to evaluates the green landscape system distribution in campus, effectively evaluate the nodes' control range, find the landscape island and makes up for the defects of landscape elements in the evaluation of campus space by spatial syntax.

\subsection{Third stage: scheme design - spatial analysis}

Scheme design is the core of planning and design. At present, most of them stay in empirical design, without parametric and quantitative evaluation of the key elements. On the basis of reinterpreting the parameters and summarizing the models, this study combines GIS spatial analysis technology and spatial syntax, according to the campus planning key elements, open space system, pedestrian road system and green landscape system are selected for evaluation.

\subsection{Fourth stage: scheme optimization - simulation optimization}

Scheme optimization is the key stage of planning and design. There is no only correct answer for optimization design. It is in pursuit of further reasonable and further improvement of design level. Spatial syntax is an effective method to predict and diagnose spatial problems because of its advantages of quantitative evaluation, but there are many limitations in its current application. On the one hand, spatial syntax is easily affected by the differences of planners' understanding in modelling and analysis. On the other hand, most of the current applications are still static evaluation, and have not gone deep into the field of planning and design to verify and improve the problems dynamically. In this study, combined with social force model, simulation technology is used to give full play to its visualization advantages, establish a typical travel behaviour simulation model, dynamically verify the accuracy of spatial syntax evaluation. In addition, according to the guidance, the simulation model is adjusted to assist the improvement and optimization of the scheme.

\section{CONCLUSIONS}

This study combines spatial syntax, GIS and social force simulation technology to improve the spatial syntax evaluation method of campus layout, interpreted the spatial syntax parameters and models from the perspective of planning, coordinate the planning and design of different majors; detailed application of space syntax in different stages of campus planning and design, integrate the social force simulation model into the post evaluation of planning and design, dynamically feedback space problems, verify its rationality and logical integrity.

To sum up, this study explored the application system of space syntax in the process of campus planning and design to a certain extent, and established a relatively perfect post evaluation system of campus planning and design.

However, 2 bottlenecks remain. First, large-scale repetitive modification is needed; second, the workload of pre-design is heavy, and the post evaluation has certain limitations. In the future, the post evaluation system will eventually change into forward design with shortening design process. The quantitative, parametric and visual evaluation system of process space syntax from the perspective of planning provides a new development opportunity for forward design, but it still needs further research on design thinking, collaborative mode and construction mode.

\section{Acknowledgments}

This work was supported in part by the scientific research project of National Ministry of Housing and Urban-Rural Construction (2017-K2-009, 2019-K-151), and the Guangxi Key Project of Research and Development (Grant No. Guike AB16380280, AB17292087), College Students' Innovative Entrepreneurial Training Plan Programme of China University of Geosciences (Beijing) (202011415233), and Guangxi postgraduate scientific research innovation project (2019YCXS120, 2020YCXS124).

\section{References}

1. Xu J, Wang B, Research of Chicago University Campus Deep Construction Based on Space Syntax Analysis. Huazhong Architecture, 10: 137139(2010).

2. Zhang Y, A Cognitive Study of Campus Spatial Morphology Based on Spatial Syntax-Take Wuhan University Campus as an Example. Architecture \& Culture, 07: 131-132(2020).

3. $\mathrm{Li} \mathrm{Z,} \mathrm{Li} \mathrm{C,} \mathrm{Li} \mathrm{C,} \mathrm{Jie} \mathrm{J,} \mathrm{An} \mathrm{algorithm} \mathrm{for} \mathrm{analysis} \mathrm{of}$ quantitative visibility in space syntax. $J X i^{\prime}$ an Univ. of Arch.\& Tech. (Natural Science Edition), 05: 716719 (2011).

4. Ma F, (2020) Spatial equity analysis of urban green space based on spatial design network analysis (sDNA): A case study of central Jinan, China, $\mathbf{6 0}$.

5. He J, Concept. Practice. Outlook-- Contemporary Campus Planning and Design. Science paper online, 7: 489 - 493(2010).

6. Gu H, Shen T, Zhou L, Chen H, Xiao F, Measuring Street Layout's Spatio -Temporal Effects on Housing Price Based on GWR and sDNA Model: The Case Study of Guangzhou. Economic Geography,03: 82 -91(2018). 